\title{
TWO-CRITERION THRESHOLD TECHNIQUES: EVIDENCE FOR SEPARATE SPATIAL AND TEMPORAL MECHANISMS?
}

\author{
Steven C. PANish ${ }^{1}$, Dan J. SWIFT ${ }^{2}$ and Robert A. SMIth ${ }^{2}$ \\ 'Vision Research Laboratory, University of New Hampshire, Durham, NH 03824, U.S.A. and \\ ${ }^{2}$ University of Michigan, Dearborn, MI 48128, U.S.A.
}

(Received 3 March 1983: in revised form 9 Mav 1983)

\begin{abstract}
Contrast thresholds were determined for counterphase flickering and drifting spatial gratings using pattern and flicker/motion criteria. In contrast to previous reports. the two criteria yielded contrast sensitivity functions (CSF) of similar form in the counterphase condition. However. moving gratings yielded CSF's of different form for the two criteria. These differences are probably due to eye movements.
\end{abstract}

Criteria Threshold Spatial frequency

\section{INTRODUCTION}

Results from several areas of research suggest a dissociation in the processing of spatial change (form) and temporal change (motion and flicker). Proposals for such a dissociation originated in the neurophysiological literature. where retinal ganglion cells were classified in accordance with their spatial summation properties (Enroth-Cugell and Robson. 1966) and response characteristics (Cleland et al. 1971). Shortly thereafter. two-mechanism hypotheses were used in the psychophysical literature to explain various phenomena including different contrast thresholds for the detection of pattern and ficker (Keesey. 1972). A common conception of such a model envisions two functionally separate mechanisms. each consisting of an ensemble of narrowly-tuned sub-mechanisms or channels (Kulikowski and Tolhurst. 1973). Much of this work has been reviewed by Legge (1978),

Two-mechanism hypotheses have generally been used to account for differences in response to stimuli which are widely separated in spatial and/or temporal frequency. Such data could result from a continuum of channels having continuously varying spatial and temporal tunings. It is not necessary to postulate functional segregation of such channels into parallel mechanisms responsible for the perception of spatial and temporal change. However, this criticism does not apply to twothreshold psychophysical procedures. where testing occurs at identical stimulus parameters. For example. Kulikowski and Tolhurst (1973) used the method-ofadjustment to measure "flicker detection" and "pattern recognition" thresholds for 0.8 and $12 \mathrm{c} / \mathrm{deg}$ sinusoidal gratings fickering sinusoidally at temporal frequencies from 0 to $15 \mathrm{~Hz}$. The contrast sensitivity functions (CSF) produced by the two thresholds were highly distinctive. pattern thresholds being low-pass. while flicker thresholds were bandpass. The thresholds were commonly separated by a factor of 2 or 3 . Signifcantly. the shapes (but not absolute sensitivities) of the pattern CSF's (plotted across temporal frequency) were identical at 0.8 and $12 \mathrm{c} / \mathrm{deg}$ as were the flicker CSF's. On the basis of these two invariant CSF's. Kulikowski and Tolhurst argued for the existence of two "systems". Harris (1980). using the same procedure with drifting gratings, found that the ratio between pattern and flicker thresholds was invariant with spatial-frequency and directly proportional to velocity. In view of this relationship he proposed velocity to be coded by the ratio of activation of separate temporal-frequency and spatial-frequency sensitive mechanisms. Harris showed that counterphase flicker could be similarly analyzed in terms of velocity-equivalent conditions and presented confirmatory two-threshold data for flickering gratings. Thus Harris demonstrated the following significant relationship: the ratio of flicker threshold to pattern threshold is invariant for a given velocity (or velocity-equivalent conditions) and varies directly with velocity. It is important to note that threshold ratios calculated from Kulikowski and Tolhurst's data obey the relationship described by Harris. although this point was not observed at the time.

Two-threshold procedures have also been used in conjunction with spatial-frequency adaptation. Tolhurst et al. (1973) adapted subjects to moving gratings and measured the temporal tuning (temporal frequency specificity) of the two hypothetical systems using motion and form thresholds. Temporal tuning was only found when using the motion detection task. which was interpreted as evidence for the twomechanisms hypothesis. Green (1981) attempted to use the two-threshold technique to evaluate the sensation following adaptation. He reported that uniform spatial criteria could not be maintained and altered the procedure to that of Watson and Nachmias (1977). Separate threshold functions were found up to $3 \mathrm{c} / \mathrm{deg}$. 
which Green interpreted as the upper limit of the transient system. Because we perceive motion at higher spatial frequencies. Green concluded that the spatial system is capable of signaling motion. It is unclear however, why the two-criterion technique should ever work if the spatial system can itself signal motion or flicker. Such a mechanism makes the dichotomy less meaningful.

Other recent experiments have obtained results which conflict with those from the two-threshold procedures. The central issue concerns whether the two criteria actually tap separate spatial and temporal mechanisms or merely reflect different signal strengths within a single multi-channel perceptual mechanism. Derrington and Henning (1981) and Virsu et al. (1982) used forced-choice procedures to compare the contrast threshold for detecting a counterphase flickering grating with thresholds for discriminating the orientation of the grating. The two functions were similar in shape over spatial and temporal frequency. Because orientation discrimination is clearly a spatial task, twomechanism models predict that it should have required more contrast at low spatial-frequency. where the spatial mechanism is proposed to be least sensitive. Burbeck (1981) used a criterion-free procedure to measure pattern and flicker thresholds in counterphasefilckered gratings. Her results differed substantially from those of the method-of-adjustment procedures. showing that the contrast threshold surface could essentially be accounted for by the pattern-threshold surface alone. Panish (1982) used a multiple-alternative forced-choice procedure to examine the appearance of near-threshold flickered gratings following adaptation to counterphase-flickered gratings. Conditions designed to tap a temporal mechanism showed that the resulting predominence of flicker ratings could be accounted for altogether by an elevated flicker false alarm rate. The important point is that in each of the above studies. the use of a less bias-prone technique has significantly reduced those experimental differences which are cited as evidence for a two-mechanism visual system.

The two-threshold procedures have often been described as different tasks, such as flicker "detection" and pattern "recognition". reflecting the presumption that different mechanisms underly them. It may be that these task differences are merely subtle criterion changes. In view of the differences between results from criterion-free/bias-correcting procedures and from method-of-adiustment experiments. it seemed prudent to perform a method-of-adjustment experiment using very carefully specified criteria representing minimal "spatial" and "temporal" percepts. Secondly. a full two-criterion spatio-temporal contrast sensitivity surface has yet to be published and studied in detail. Finally. despite the common assumption that motion and counterphase flicker are largely equivalent. we decided to measure two such surfaces. one for moving and one for flickering gratings. so that the results could be compared.

\section{METHODS}

\section{Stimuli}

Counterphase-flickered. vertically oriented sinewave gratings were generated using a Xitan microcomputer equipped with an analog interface designed in this lab. The display was a Hewlett-Packard 1332A. viewed at $75 \mathrm{~cm}$. Mean luminance was $35 \mathrm{~cd} \mathrm{\textrm {m } ^ { 2 }}$. Test gratings occupied the center $2.5 \mathrm{deg}$ of a $10 \mathrm{deg}$ wide display and had a 2 deg wide. mean luminance border on either side. The grating extended the full $8 \mathrm{deg}$ vertical height of the display. The center of the field contained a fixation point. Twenty-four combinations of spatial and temporal frequencies were used. 0.5. 1. 4. and $8 \mathrm{c} / \mathrm{deg}$. and 0.5 . 1. 2. 4. 8. and $16 \mathrm{~Hz}$ counterphase. Subject P.C. was also run at $2 \mathrm{c}$ deg.

\section{Subjects}

One of the authors. S.P.. a 29-yr old corrected myope, and P.C., a 34-yr old psychophysically naive male with normal acuity. served as subjects.

\section{Procedure}

The subject communicated with the computer via a movable keyboard. On each trial a $6 \mathrm{sec}$ blank field was presented. immediately followed by a $6 \mathrm{sec}$ test grating presentation. During the latter period. test grating contrast was adjusted by the subject. When setting a pattern threshold, the subject adjusted the contrast either until periodic bars or vertical spatial structure was just visible. When setting a flickermotion threshold. contrast was adjusted until movement or flicker was just observed. The condition was terminated when seven thresholds had been set. Subiect S.P. ran each condition in two or three experimental sessions yielding 14 or 21 threshold settings respectively. Subject P.C. ran each condition in 3 or 4 sessions, yielding 21 or 28 settings respectively.

\section{RESUI.TS}

\section{Counterphase-flicker}

Thresholds are plotted in Figs 1 and 2. Several striking differences are immediately apparent between these data and those of Kulikowski and Tolhurst (1973). Perhaps the most important is that the shapes of the CSF's are generally very similar for the pattern and flicker thresholds at any given spatial-frequency. At 8 and $4 \mathrm{c} / \mathrm{deg}$ both functions are low-pass. At 1 and $0.5 \mathrm{c} / \mathrm{deg}$ the curves show more bandpass character. The curve-shapes are consistently similar over temporal frequency for subject S.P.. while at $0.5 \mathrm{c} / \mathrm{deg}$ the pattern and flicker threshold functions are different for subject P.C. It is also notable that our data generally show less than a factor of two separation of the form and flicker CSF's.

As spatial frequency was increased. crossover points between the pattern and flicker/motion threshold functions migrated to higher temporal frequency. However. the ratios of the two thresholds were not constant 

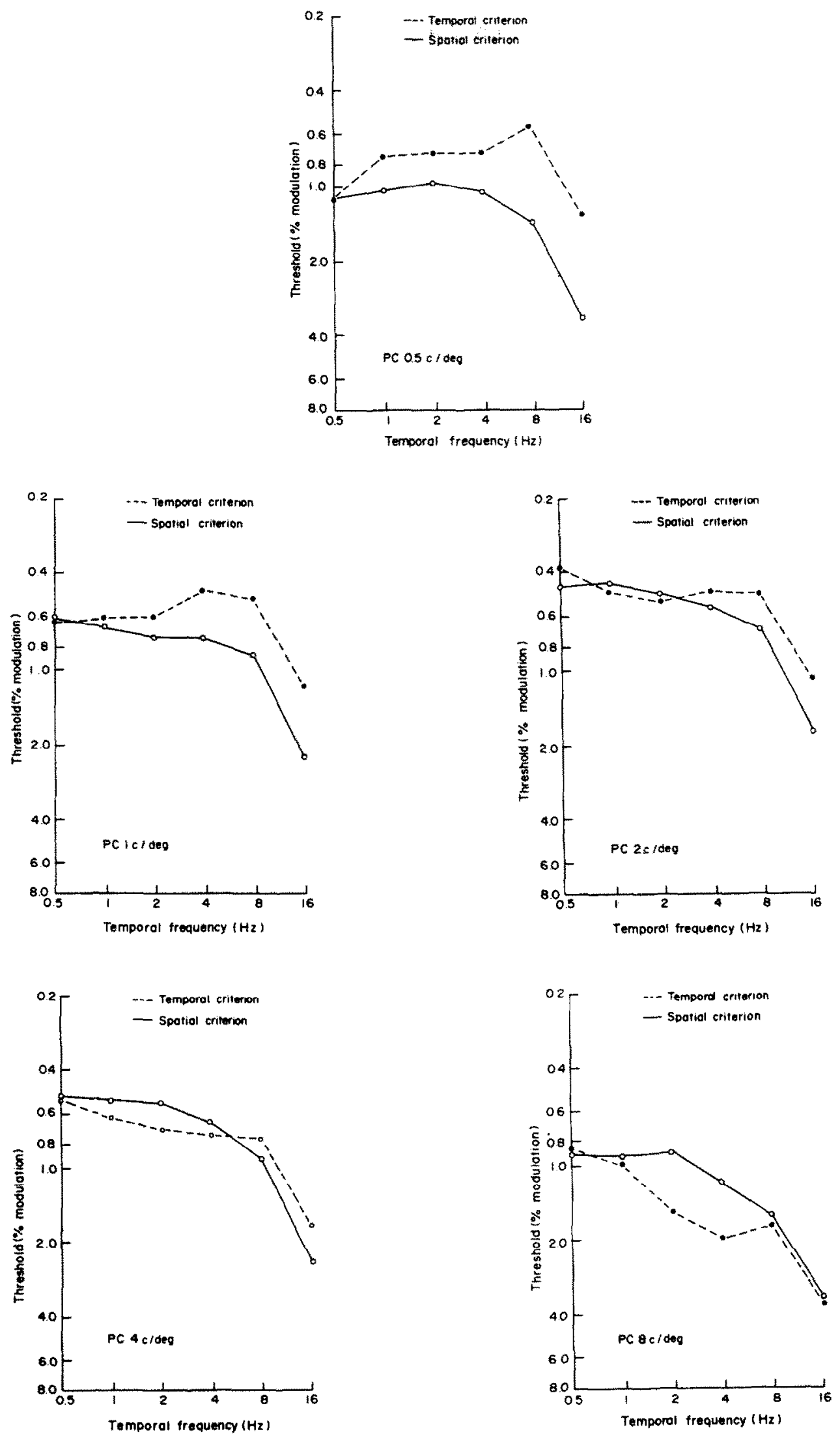

Fig. 1. Temporal and spatial thresholds for counterphase flickered gratings. Subject P.C. 's standard crrors are not shown as virtually all were smaller than the data points. 

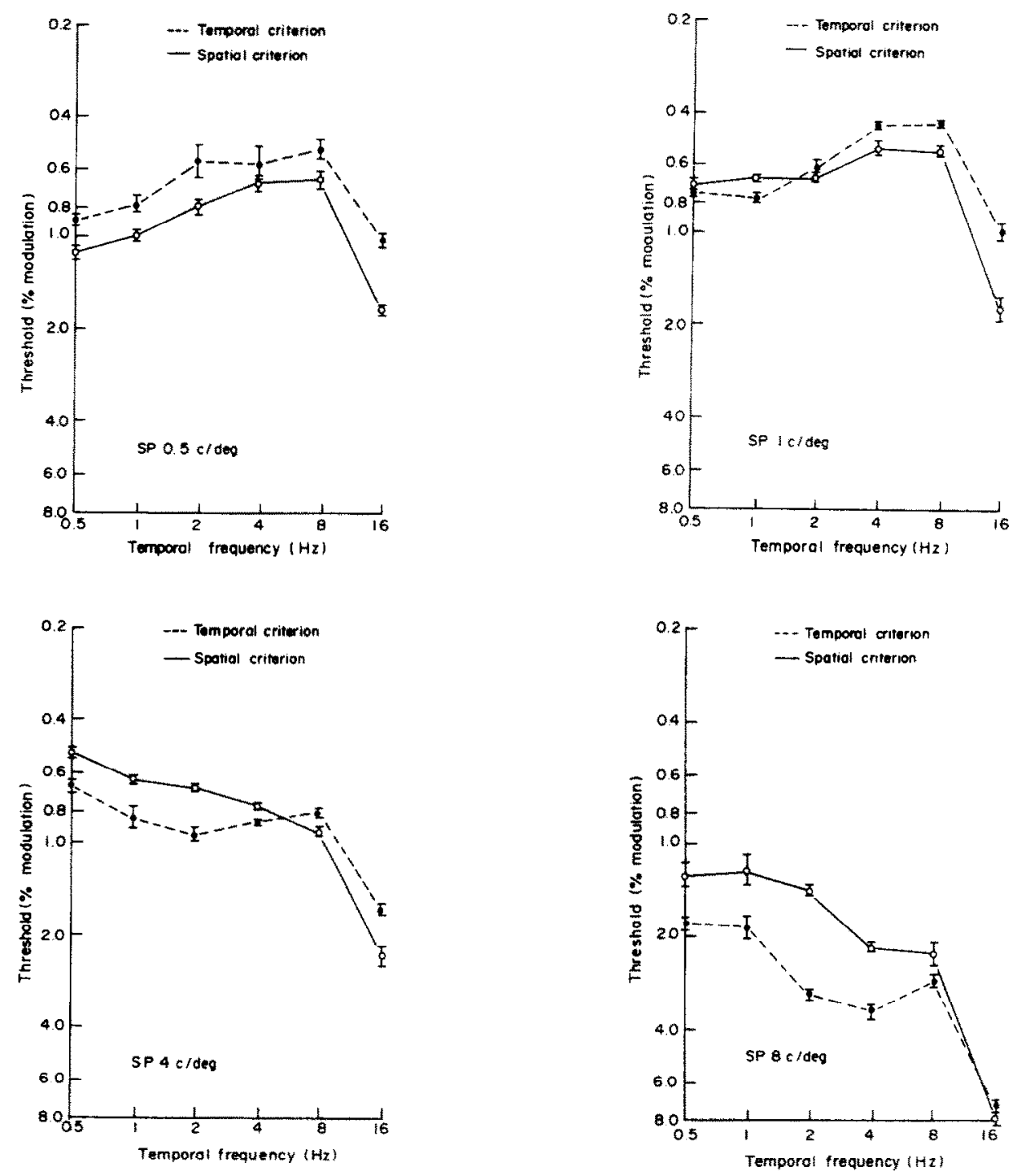

Fig. 2. Temporal and spatial thresholds for counterphase flickered gratings. Subject S.P.

for velocity-equivalent conditions. nor did they increase linearly or even monotonically with temporal frequency as did those of Harris (1980). These ratios do not appear to offer a basis for the coding of velocity. Although these data show reliably separable pattern and flicker thresholds. the generally small separation and similar form of the resultant curves resemble criterion shifts rather than separate mechanisms.

\section{Moving gratings}

Notable differences exist between the temporal response functions for moving gratings and those generated by counterphase flickering gratings. Thresholds are plotted in Figs 3 and 4 . These data more closely resemble the counterphase data of Kulikowski and Tolhurst (1973) than do our own counterphase data. This resemblance is due to the

Table 1. The ratio of the threshold for seeing flicker to the threshold for seeing pattern in counterphase flickered gratings. Subiect P.C.

\begin{tabular}{|c|c|c|c|c|c|c|}
\hline \multirow{2}{*}{ Spatial frequency (c/deg) } & \multicolumn{6}{|c|}{ Temporal frequency. HZ counterphase } \\
\hline & 0.5 & 1 & 2 & 4 & 8 & 16 \\
\hline $\begin{array}{l}\text { Spatial irequency (c/oeg) } \\
0.5\end{array}$ & 0.86 & 0.88 & 0.69 & 0.59 & 0.36 & 0.35 \\
\hline 1 & 1.06 & 0.92 & 0.83 & 0.65 & 0.59 & 0.52 \\
\hline 2 & 0.85 & 1.09 & 1.08 & 0.86 & 0.72 & 0.62 \\
\hline 4 & 1.05 & 1.20 & 1.29 & 1.11 & 0.82 & 0.73 \\
\hline 8 & 0.96 & 1.08 & 0.56 & 0.60 & 1.11 & 1.05 \\
\hline
\end{tabular}



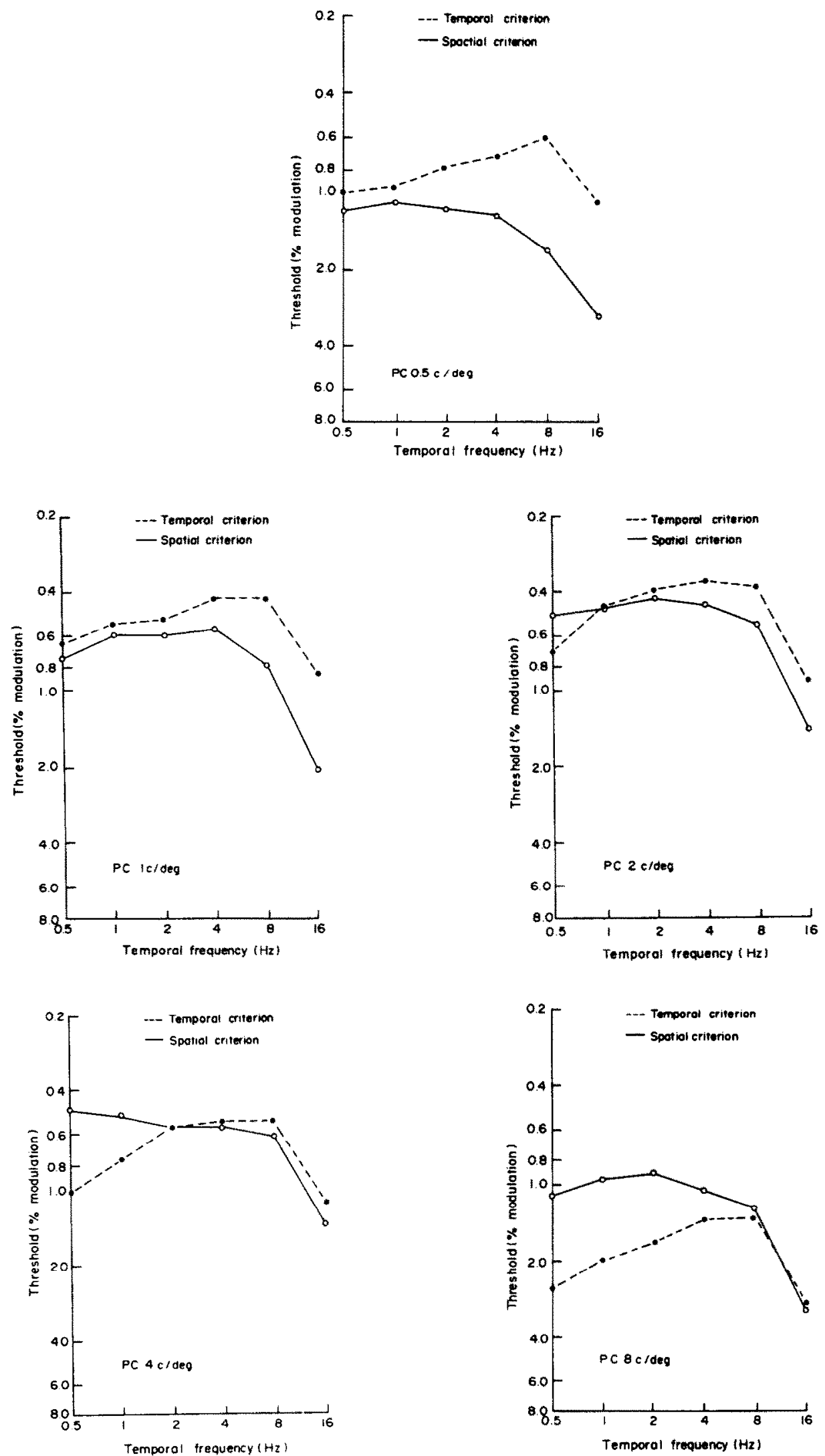

Fig. 3. Temporal and spatial thresholds for drifting gratings. Subject P.C. Standard errors were generally smaller than the data points. 

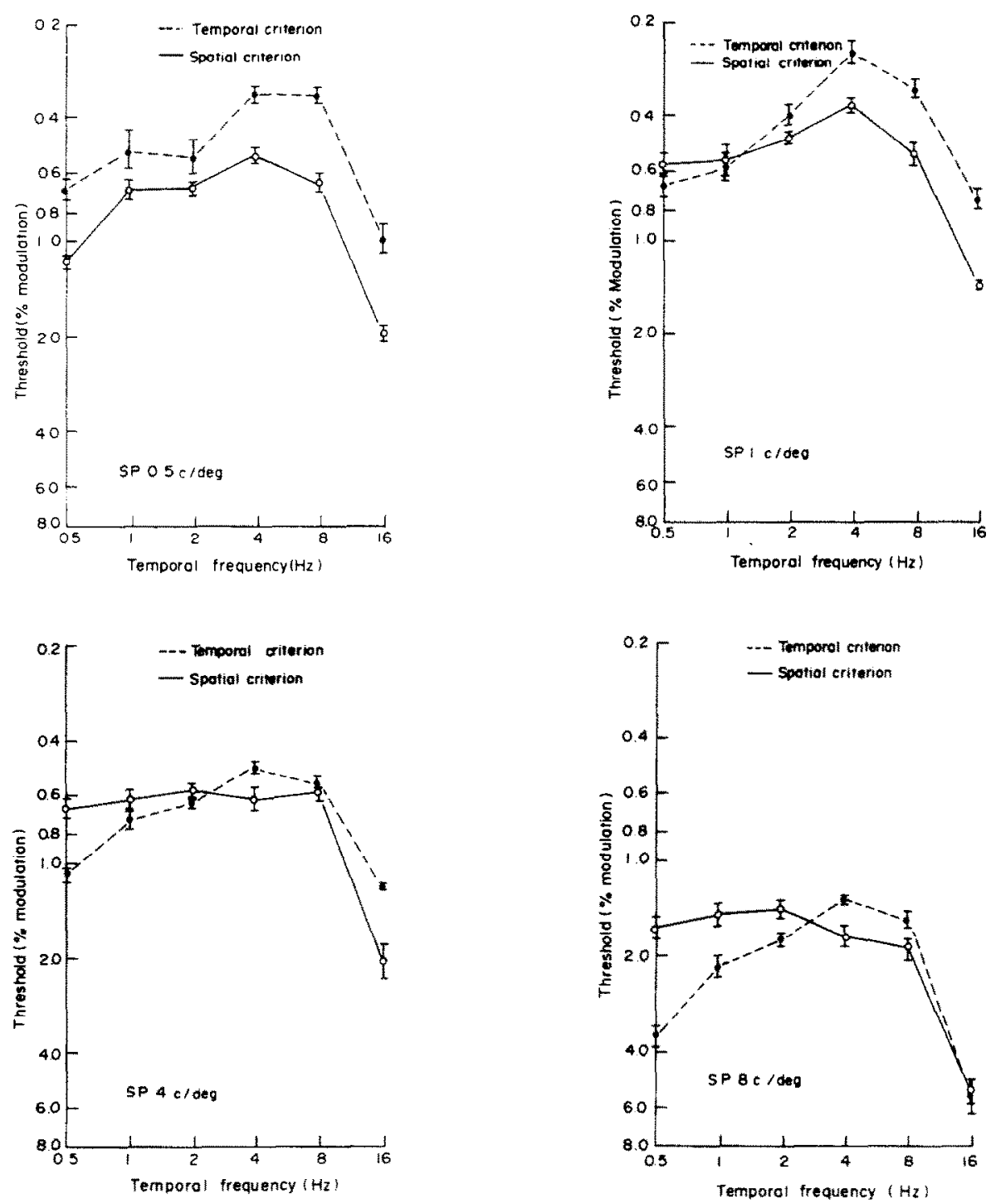

Fig. 4. Temporal and spatial thresholds for drifting gratings. Subject S.P.

relative invariance of the forms of the temporal response functions across spatial frequency. In all cases. flicker CSF's show a clear bandpass shape. while the pattern CSF's are predominantly low pass except at low spatial frequency. Crossover points of the two functions rise with spatial frequency. Ratios of the motion to pattern thresholds vary monotonically with spatial frequency and inversely with temporal frequency (Table 2). The ratios did not vary linearly across spatial-frequency as described by Harris (1980). but as a power law with an exponent in the range of $0.3-0.5$.

Table 2. The ratio of the threshold for seeing motion to the threshoid for seeing pattern in drifting gratings. Eyual velocities lie along the diagonals of the table. Subject P.C.

\begin{tabular}{|c|c|c|c|c|c|c|}
\hline \multicolumn{7}{|c|}{ Temporal frequency. $\mathrm{HZ}$} \\
\hline & 0.5 & 1 & 2 & 4 & 8 & 16 \\
\hline \multicolumn{7}{|c|}{ Spatial frequency (c/deg) } \\
\hline 0.5 & 0.99 & 0.73 & 0.75 & 0.69 & 0.40 & 0.30 \\
\hline 1 & 0.88 & 0.90 & 0.87 & 0.75 & 0.54 & 0.41 \\
\hline 2 & 1.38 & 0.98 & 0.93 & 0.80 & 0.71 & 0.64 \\
\hline 4 & 2.13 & 1.47 & 1.02 & 0.96 & 0.87 & 0.81 \\
\hline 8 & 2.35 & 2.13 & 1.88 & 1.31 & 1.10 & 0.92 \\
\hline
\end{tabular}




\section{DISCUSSION}

It is clear that subjects can consistently set separate thresholds for the perception of temporal and spatial variation, and that there is a general correspondence between data from different subjects. However. our data present several problems: (1) why do we find significant differences between drifting and counterphase gratings. and can either condition be regarded as more fundamental. explaining the other. (2) why do our results differ from those of earlier experiments. (3) can these results support a two mechanism model of spatial and temporal vision?

No obvious transform relates the results obtained from the drifting and counterphase conditions. They may represent different visual processes. However. we speculate that the differences between the two data sets result from two factors: the use of different visual strategies for optimizing the desired percepts, and the difficulty of avoiding tracking with drifting gratings. Kelly (1977) has demonstrated that the form of threshold functions for unstabilized counterphase gratings closely approximates results obtained with stabilization. In contrast, experiments which use drifting gratings are more prone to artifacts resulting from involuntary eye-movements (tracking). Since tracking affects the temporal parameters of the retinal image, it is an important factor in the measurement of thresholds. This is particularly true at high spatial frequencies. where small variations in velocity produce large changes in temporal frequency on the retina (Arend. 1976). The net effect of tracking is to reduce the effective temporal frequency of the stimulus. Sensitivity decreases at high temporal frequency. so reduction of the effective temporal frequency by tracking would result in less high temporal frequency fall-off, flattening the CSF. Such flattening should be maximal for high spatial-frequency drifting gratings. This may be observed clearly in the pattern threshold functions and somewhat less so in the motion threshold functions. This difference may result from use of different patterns of eye-movement in the two tasks, such that adopting a pattern criterion results in more tracking than does a motion criterion.

The results from the counterphase condition in this study differ substantially from those of Kulikowski and Tolhurst (1973) and Harris (1980). but are very similar to those from the two method-of-adjustment conditions of Burbeck (1981). Pattern and flicker thresholds obtained in the present study are clearly separable. but we feel that the modest degree of separability we observe could result solely from criterion shifts. without involvement of separate mechanisms. On the other hand. the striking invariances reported by Kulikowski and Tolhurst (1973) and Harris (1980) are unlikely to have resulted from experimental error. and clearly require an explanation. Until a good reconciliation can be found for these discrepancies. we feel that our results. together with the results of Derrington and Henning (1981). Burbeck (1981) and Panish (1982). suggest the need for caution in the use of twocriterion techniques for postulating separate pattern and flicker mechanisms.

Acknowledgements - This project was supported by AFOSR research grant $80-0045$ and University of New Hampshire CURF grant S-216.

\section{REFERENCES}

Arend L. (1976) Temporal determinants of the form of the spatial contrast threshold MTF. Vision Res. 16. 1035 1402.

Burbeck C. (1981) Criterion-free pattern and flicker thresholds, J. Opt. Soc, Am. 71. 1343-1350.

Cleland B. G.. Dubin M. W. and Levick W. R. (1971) Sustained and transient neurons in the cat's retina and lateral geniculate nucleus. J. Physiol. 217. 473-496.

Derrington A. and Henning G. (1981) Pattern discrimination with flickering stimuli. Vision Res. 21, 597-602.

Enroth-Cugell C. S. and Robson J. (1966) The contrast sensitivity of retinal ganglion cells of the cat. $J$. Physiol. 187, 517-552.

Green M. (1981) Psychophysical relationships among mechanisms sensitive to pattern. motion. and ficker. Vision Res.. 21. 971-983.

Harris M. G. (1980) Velocity specificity of the flicker to pattern sensitivity ratio in human vision. Vision Res. 20 . 687-691.

Kelly D. H. (1977) Visual contrast sensitivity. Optical Acta 24, 107-129.

Kulikowski J. and Tolhurst D. (1973) Psychophysical evidence for sustained and transient detectors in human vision. J. Physiol. 232. 149-162.

Legge G. (1978) Sustained and transient mechanisms in human vision: Temporal and spatial properties. Vision Res. 18. 69-81.

Panish S. (1982) Time-course of spatio-temporal adaptation: with respect to the existence of separate spatial and temporal visual mechanisms. Unpublished Doctoral Dissertation. Univ. of New Hampshire.

Tolhurst D.. Sharpe C. and Hart G. (1973). The analysis of the drift rate of moving sinusoidal gratings. Vision Res. 13, 2545-2555.

Tulunay Keesey U. (1972) Flicker and Pattern Detections: a comparison of thresholds. J. opt. Soc. Am. 62. 446448.

Virsu V.. Rovamo J.. Laurinen P. and Nasanen R. (1982) Temporal contrast sensitivity and cortical magnification. Vision Res. 22. 1211-1217.

Watson A. and Nachmias J. (1977) Patterns of temporal interaction in the detection of gratings. Vision Res. 17. 893-902. 